\title{
Changes in Soft Tissue Parameters Following Free Gingival Grafts Around Implants: A Prospective Cohort Study
}

UNIVERSITY of MARYLAND SCHOOL OF DENTISTRY

\author{
Se-Lim Oh, Chao Ji, and Thomas Oates \\ Department of Advanced Oral Science and Therapeutics \\ University of Maryland School of Dentistry
}

\section{Introduction}

Implants exhibiting minimal keratinized mucosa (KM) display increased mucosal inflammation, which may contribute to crestal bone loss. There is limited data regarding changes in the width of $\mathrm{KM}$ and the level of mucosal margin (MM) following free gingival grafts (FGGs) around implants. The aims of this study were to validate Image J measurements by comparing those with clinical evaluation, and to examine the changes in the width of KM and the level of MM following FGGs around implants with ImageJ measurements.

\section{Materials and Methods}

18 implants in 10 subjects who received FGGs were included in this study. The width of KM from MM to the mucogingival junction and the level of MM in relation to the implant crown margin were measured at baseline, 6 and 18 months after FGGs with Colorvue $^{\circledR} 12$ probe, and corresponding clinical photographs were taken.

The width of KM and MR were measured on the images by CJ who was blinded to research subjects and clinical evaluation. Pearson's $r$ and Bland-Altman plots were used to investigate the strength of relationship and the agreements between the clinical and the ImageJ measurements. A one-way repeated measures ANOVA followed by a post-hoc test was used to examine changes in the width of $\mathrm{KM}$ and MR.

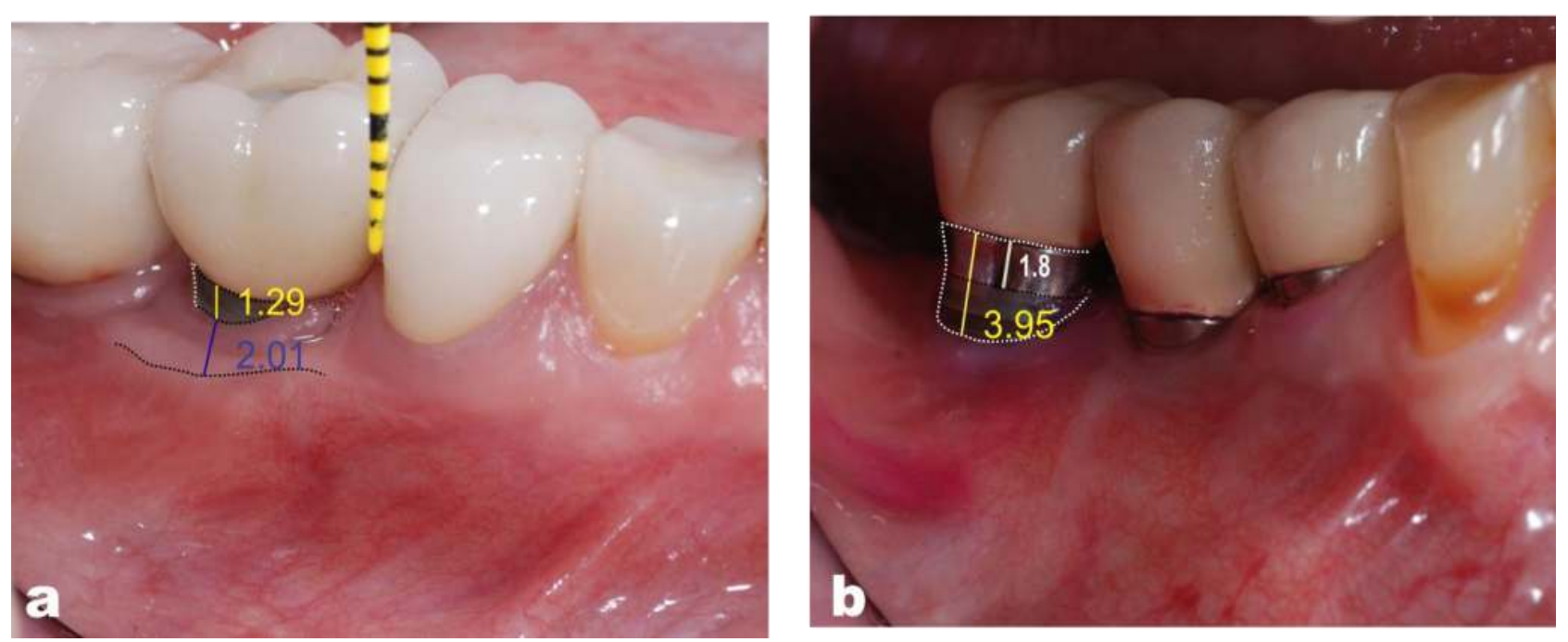

Figure 1. Examples of the image measurements $(\mathrm{mm})$. (a) The scale for the photograph was set with the probe placed. (b) The scale for the photograph was set with the known implant collar dimension.

\section{Results}

One male and nine females received FGGs around a total of 18 single implants. The mean implant survival was $4 \pm 2.8$ years at baseline. Figure 2 shows two examples of cases in this study.

The correlation was significant between ImageJ and clinical evaluation (Table 1).

Table 1. Comparisons between ImageJ measurements and clinical evaluation. KM, keratinized mucosa. MR: mucosal recession.

${ }^{* *}$ Correlation is significant at the 0.01 level (2-tailed).

$\begin{array}{cccc} & \text { Measurements } & \text { Mean } \pm S D & \text { Correlation } \\ \text { KM } & \text { Clinical evaluation }(n=54) & 2.12 \pm 1.8 & \mathrm{r}=.96^{* *} \\ & \text { ImageJ }(\mathrm{n}=54) & 2.25 \pm 1.7 & \mathrm{p}=.0005 \\ \text { MR } & \text { Clinical evaluation }(\mathrm{n}=54) & 1.21 \pm 1.04 & \mathrm{r}=.94^{* *} \\ & \text { ImageJ }(\mathrm{n}=54) & 1.02 \pm 0.97 & \mathrm{p}=.0005\end{array}$
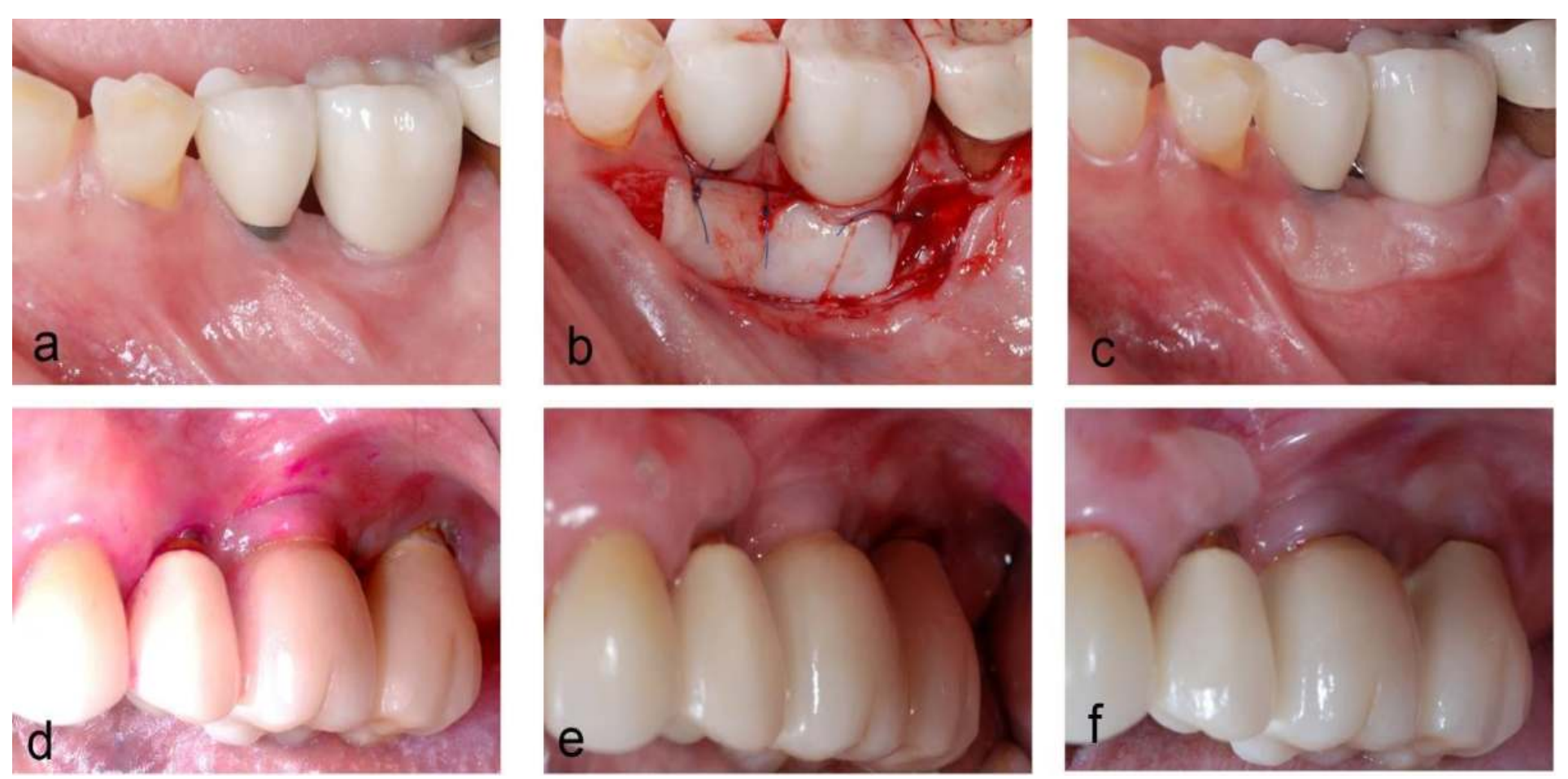

Figure 2. Case 1. (a) Baseline of \#19 and \#20 implant. (b) FGG surgery. (c) 6 months after FGG. Case 2. (d) Baseline of \#13 and \#15 implant. (e) 6 months after FGGs. (f) 18 months after FGGs.

The Bland-Altman plots showed good agreements between two measurement methods (Figure 3).

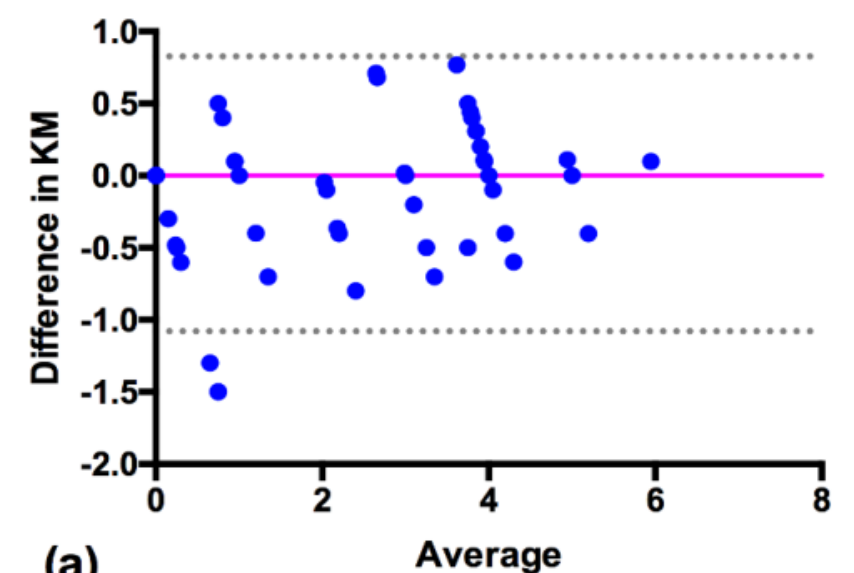

(a)

Average

(b)

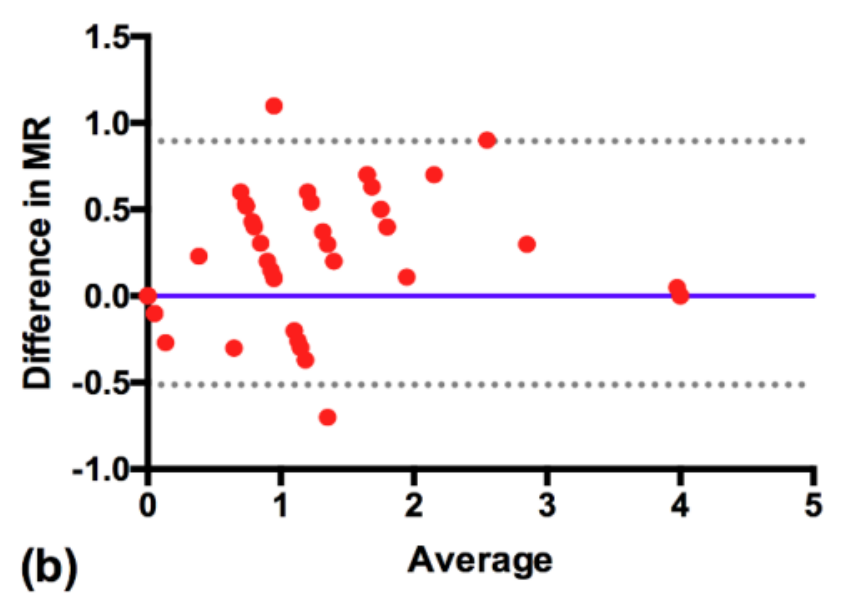

Figure 3. Bland-Altman plots for the agreements between ImageJ measurements and clinical evaluation. (a) Difference against the width of KM. (b) Difference against MR by clinical and ImageJ measurements.

There was a significant gain in $\mathrm{KM}$ width by $2.8 \pm 0.8 \mathrm{~mm}$ at 18 months from $0.5 \pm 0.6 \mathrm{~mm}$ at baseline. The mean MR was $1.3 \pm 0.9$ $\mathrm{mm}$ at baseline. There was a significant reduction in MR at 6 and 18 months (Figure 4).

Further, there were a significant gain in $\mathrm{KM}$ and a significant reduction in MR between 6 and 18 months after FGGs. Total coverages were observed in 6 out of 8 cases with implant thread exposure at 18 months following FGGs.
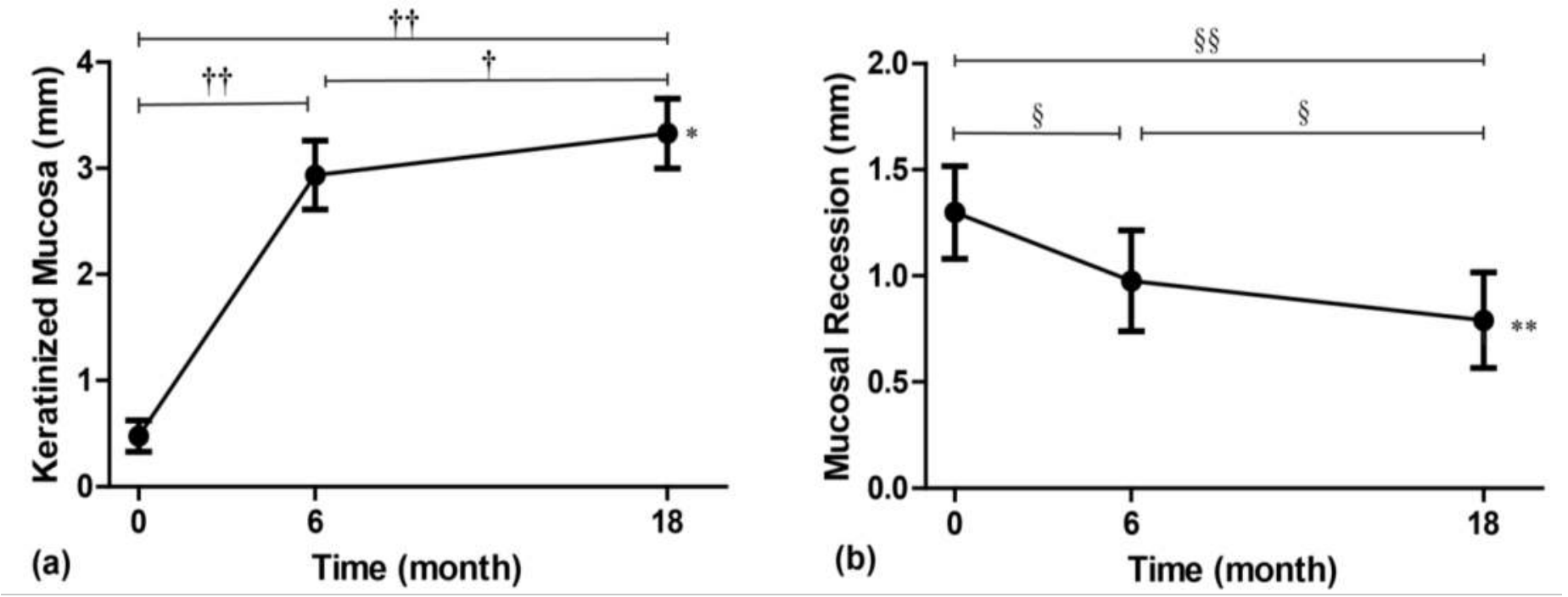

Figure 4. Changes in the width of $K M$ and the level of MM (mean $\pm S E M$ ). (a) Changes in KM. *(one-way repeated measures ANOVA, $\mathrm{F}=73.379, \mathrm{p}=.0005) \dagger$ $\mathrm{p}=.001$, t† $\mathrm{p}=.0005$ (Bonferroni's multiple comparisons). (b) The distance from the crown margin to mucosal margin. ${ }^{* *}$ (the one-way repeated measures ANOVA, $\mathrm{F}=12.589, \mathrm{p}=.0005)$. $\S p<.05$, $\S \S \mathrm{p}<.01$ (Bonferroni's multiple comparisons) in comparison among each time point.

\section{Conclusion}

- The ImageJ analysis is reliable and the measurements between ImageJ and clinical evaluation are interchangeable.

- In conjunction with gains in the width of $\mathrm{KM}$, postoperative coronal migration of the keratinized mucosal tissue occurred following FGGs around implants. 